hep-ph/9301285

IEM-FT-67/93

January 1993

\title{
The electroweak phase transition with a singlet *
}

\author{
J.R. Espinosa ${ }^{\dagger}$ and M. Quirós \\ Instituto de Estructura de la Materia. CSIC \\ Serrano, 123 E-28006 Madrid. Spain
}

\begin{abstract}
We study the electroweak phase transition in the minimal extension of the Standard Model: an extra complex singlet with zero vacuum expectation value. The first-order phase transition is strengthened by the cubic term triggered in the one-loop effective potential by the extra boson. Plasma effects are considered to leading order: they shield the cubic terms and weaken the first-order phase transition. We find a region in the parameter space where baryon asymmetry washout is avoided for Higgs masses consistent with present experimental bounds. However in that region the theory becomes non-perturbative for scales higher than $10^{10} \mathrm{GeV}$.
\end{abstract}

*Work partly supported by CICYT under contract AEN90-0139.

${ }^{\dagger}$ Supported by Comunidad de Madrid Grant. 
The discovery that the Standard Model of the electroweak interactions contains an anomaly which violates $B+L$ [四], and that the rate of $B+L$ violation is unsuppressed at high temperatures [2], has revived interest in the possibility of creating the baryon asymmetry at the electroweak phase transition (EWPT) [3]. In particular, the condition that the baryon excess generated at the EWPT is not washed out requires a strong enough first-order

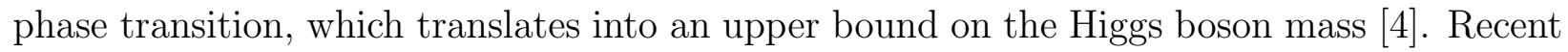
analyses of the minimal Standard Model (MSM) at one-loop [5], and including plasma effects [6, 7] in various approximations [8]-[14], show that the above upper bound is inconsistent with the present experimental lower bound [15], i.e. that the phase transition is not strongly enough first order. Though, in our opinion, this issue is not yet fully settled it is interesting to study extensions of the MSM where the phase transition can be made consistent with present experimental lower bounds on the Higgs boson mass.

In this letter we study the phase transition in the simplest of these extensions, which consists in adding to the MSM a complex gauge singlet with zero vacuum expectation value. This extension was proposed in refs. [5, 16] as the simplest way of overcoming the problems encountered in the MSM. In fact the added boson generates a cubic term in the one-loop effective potential, which can trigger a strong first-order phase transition if it is not shielded by a heavy $S U(2) \times U(1)$ invariant mass 1 . However, as noticed in ref. [18], the extra boson behaves as the longitudinal components of $W$ and $Z$ gauge bosons and the corresponding cubic term can also be shielded by plasma effects. Fortunately we will see this is not always the case and find the region in parameter space where the phase transition is strong enough first order for values of the Higgs boson mass beyond the experimental bounds.

The lagrangian of the model is defined as:

$$
\mathcal{L}=\mathcal{L}_{M S M}+\partial^{\mu} S^{*} \partial_{\mu} S-M^{2} S^{*} S-\lambda_{S}\left(S^{*} S\right)^{2}-2 \zeta^{2} S^{*} S H^{*} H
$$

where $H$ is the MSM doublet with $\langle H\rangle=\phi / \sqrt{2}, \phi$ is the classical field, and $M^{2}, \lambda_{S}, \zeta^{2} \geq 0$, to guarantee that $\langle S\rangle=0$ at all temperatures $\mathrm{Q}$.

The tree-level potential is that of the MSM

$$
V_{\text {tree }}=-\frac{\mu^{2}}{2} \phi^{2}+\frac{\lambda}{4} \phi^{4}
$$

and the fields contributing to the effective potential are those of the MSM, i.e. the Higgs field $h$, the Goldstone bosons $\vec{\chi}$, the gauge bosons $W^{ \pm}, Z, \gamma$ and the top quark $t$, with masses

$$
\begin{gathered}
m_{h}^{2}(\phi)=3 \lambda \phi^{2}-\mu^{2}, \quad m_{\chi}^{2}=\lambda \phi^{2}-\mu^{2} \\
m_{W}^{2}(\phi)=\frac{g^{2}}{4} \phi^{2}, \quad m_{Z}^{2}(\phi)=\frac{g^{2}+g^{\prime 2}}{4} \phi^{2}, \quad m_{\gamma}=0 \\
m_{t}^{2}(\phi)=\frac{h_{t}^{2}}{2} \phi^{2}
\end{gathered}
$$

\footnotetext{
${ }^{1}$ This result also holds when the gauge singlet acquires a vacuum expectation value. This case has been recently analyzed at the one-loop level in ref. [17], where plasma effects are not considered.

${ }^{2} \mathrm{~A}$ cubic term in (11) would destabilize the potential along some direction in the $S$ plane for $\lambda_{S}=0$. We assume a global $U(1)$ symmetry $S \rightarrow e^{i \alpha} S$ which prevents the appearance of such cubic term.
} 
(where $g, g^{\prime}, h_{t}$ are the $S U(2) \times U(1)$ gauge and top Yukawa couplings, respectively), and the $S$ boson, with a mass

$$
m_{S}^{2}(\phi)=M^{2}+\zeta^{2} \phi^{2} .
$$

The temperature dependent effective potential can be calculated using standard techniques [6]. Plasma effects in the leading approximation can be accounted by the one-loop effective potential improved by the daisy diagrams [6, 7]. Imposing renormalization conditions preserving the tree level values of $v^{2} \equiv \mu^{2} / \lambda$, and working in the 't Hooft-Landau gauge, the $\phi$-dependent part of the effective potential can be written in the high-temperature expansion as

$$
V_{\text {eff }}(\phi, T)=V_{\text {tree }}+\Delta V_{B}+\Delta V_{F}
$$

where

$$
\begin{gathered}
\Delta V_{B}=\sum_{i=h, \chi, W_{L}, Z_{L}, \gamma_{L}, W_{T}, Z_{T}, \gamma_{T}, S} g_{i} \Delta V_{i} \\
\Delta V_{i}=\left\{\frac{m_{i}^{2}(\phi) T^{2}}{24}-\frac{\mathcal{M}_{i}^{3}(\phi) T}{12 \pi}-\frac{m_{i}^{4}(\phi)}{64 \pi^{2}}\left[\log \frac{m_{i}^{2}(v)}{c_{B} T^{2}}-2 \frac{m_{i}^{2}(v)}{m_{i}^{2}(\phi)}+\delta_{i \chi} \log \frac{m_{h}^{2}(v)}{m_{i}^{2}(v)}\right]\right\},
\end{gathered}
$$

where the last term comes from the infinite running of the Higgs mass from $p^{2}=0$ to $p^{2}=m_{h}^{2}$ and cancels the logarithmic infinity from the massless Goldstone bosons at the zero temperature minimum, and

$$
\Delta V_{F}=g_{t}\left\{\frac{m_{t}^{2}(\phi) T^{2}}{48}+\frac{m_{t}^{4}(\phi)}{64 \pi^{2}}\left[\log \frac{m_{t}^{2}(v)}{c_{F} T^{2}}-2 \frac{m_{t}^{2}(v)}{m_{t}^{2}(\phi)}\right]\right\}
$$

The number of degrees of freedom $g_{i}$ in (6,8) are given by

$$
\begin{gathered}
g_{h}=1, g_{\chi}=3, g_{S}=2, g_{t}=12 \\
g_{W_{L}}=g_{Z_{L}}=g_{\gamma_{L}}=1, g_{W_{T}}=g_{Z_{T}}=g_{\gamma_{T}}=2
\end{gathered}
$$

while the coefficients $c_{B}$ and $c_{F}$ in (7,8) are defined by: $\log c_{B}=3.9076, \log c_{F}=1.1350$.

The masses $m_{i}^{2}(\phi)$ in (7,8) are defined in (3,4) and the Debye masses $\mathcal{M}_{i}^{2}$ in (7) for $i=h, \chi, S, W_{L}, W_{T}, Z_{T}, \gamma_{T}$ are

$$
\mathcal{M}_{i}^{2}=m_{i}^{2}(\phi)+\Pi_{i}(\phi, T)
$$

where the self-energies $\Pi_{i}(\phi, T)$ are given by

$$
\begin{gathered}
\Pi_{h}(\phi, T)=\left(\frac{3 g^{2}+g^{\prime 2}}{16}+\frac{\lambda}{2}+\frac{h_{t}^{2}}{4}+\frac{\zeta^{2}}{6}\right) T^{2} \\
\Pi_{\chi}(\phi, T)=\left(\frac{3 g^{2}+g^{\prime 2}}{16}+\frac{\lambda}{2}+\frac{h_{t}^{2}}{4}+\frac{\zeta^{2}}{6}\right) T^{2} \\
\Pi_{S}(\phi, T)=\frac{\lambda_{S}+\zeta^{2}}{3} T^{2} \\
\Pi_{W_{L}}(\phi, T)=\frac{11}{6} g^{2} T^{2}
\end{gathered}
$$




$$
\Pi_{W_{T}}(\phi, T)=\Pi_{Z_{T}}(\phi, T)=\Pi_{\gamma_{T}}(\phi, T)=0
$$

The Debye masses $\mathcal{M}_{i}^{2}$ for $i=Z_{L}, \gamma_{L}$ are given by

$$
\left(\begin{array}{cc}
\mathcal{M}_{Z_{L}}^{2} & 0 \\
0 & \mathcal{M}_{\gamma_{L}}^{2}
\end{array}\right)=R\left(\theta_{W}^{(1)}\right)\left(\begin{array}{cc}
m_{Z}^{2}(\phi)+\Pi_{Z_{L} Z_{L}} & \Pi_{Z_{L} \gamma_{L}} \\
\prod_{\gamma_{L} Z_{L}} & \Pi_{\gamma_{L} \gamma_{L}}
\end{array}\right) R^{-1}\left(\theta_{W}^{(1)}\right)
$$

with the rotation $R\left(\theta_{W}^{(1)}\right)$

$$
R\left(\theta_{W}^{(1)}\right)=\left(\begin{array}{cc}
\cos \theta_{L}^{(1)} & -\sin \theta_{L}^{(1)} \\
\sin \theta_{L}^{(1)} & \cos \theta_{L}^{(1)}
\end{array}\right)
$$

and the self-energies f $^{3}$

$$
\begin{gathered}
\Pi_{Z_{L} Z_{L}}(\phi, T)=\left(\frac{2}{3} g^{2} \cos ^{2} \theta_{W}+\frac{1}{6} \frac{g^{2}}{\cos ^{2} \theta_{W}}\left(1-2 \sin ^{2} \theta_{W} \cos ^{2} \theta_{W}\right)\right. \\
\left.+\frac{g^{2}}{\cos ^{2} \theta_{W}}\left(1-2 \sin ^{2} \theta_{W}+\frac{8}{3} \sin ^{4} \theta_{W}\right)\right) T^{2} \\
\Pi_{\gamma_{L} \gamma_{L}}(\phi, T)=\frac{11}{3} e^{2} T^{2} \\
\prod_{\gamma_{L} Z_{L}}(\phi, T)=\frac{11}{6} e g \frac{\cos ^{2} \theta_{W}-\sin ^{2} \theta_{W}}{\cos \theta_{W}} T^{2}
\end{gathered}
$$

The angle $\theta_{L}^{(1)}$ in (ㅍ) is the one-loop temperature dependent correction to the electroweak angle. In fact the angle $\theta_{L}(\phi, T)$ defined by

$$
\theta_{L}(\phi, T)=\theta_{W}+\theta_{L}^{(1)}
$$

maps $\left(A_{3}, B\right)$ into $(Z, \gamma)$

Using $(1820)$ one obtains the eigenvalues and rotation angle in (10) as:

$$
\begin{gathered}
\mathcal{M}_{Z_{L}}^{2}=\frac{1}{2}\left[m_{Z}^{2}(\phi)+\frac{11}{6} \frac{g^{2}}{\cos ^{2} \theta_{W}} T^{2}+\Delta(\phi, T)\right] \\
\mathcal{M}_{\gamma_{L}}^{2}=\frac{1}{2}\left[m_{Z}^{2}(\phi)+\frac{11}{6} \frac{g^{2}}{\cos ^{2} \theta_{W}} T^{2}-\Delta(\phi, T)\right] \\
\quad \sin 2 \theta_{L}^{(1)}(\phi, T)=-\frac{2 \prod_{\gamma_{L} Z_{L}}}{\Delta} \\
\sin 2 \theta_{L}(\phi, T)=\sin 2 \theta_{W} \frac{m_{Z}^{2}(\phi)}{\Delta(\phi, T)}
\end{gathered}
$$

with

$$
\Delta^{2}(\phi, T)=m_{Z}^{4}(\phi)+\frac{11}{3} \frac{g^{2} \cos ^{2} 2 \theta_{W}}{\cos ^{2} \theta_{W}}\left[m_{Z}^{2}(\phi)+\frac{11}{12} \frac{g^{2}}{\cos ^{2} \theta_{W}} T^{2}\right] T^{2}
$$

\footnotetext{
${ }^{3}$ We correct the coefficients of the last terms in (18), $\frac{8}{3}$, and in (19), $\frac{11}{3}$, which were misprinted as 4 and $\frac{15}{3}$, respectively, in 10 .
} 
It is clear from (25,26) that at zero temperature the electroweak angle coincides with the usual one: $\Delta(\phi, 0)=m_{Z}^{2}(\phi), \theta_{L}(\phi, 0) \equiv \theta_{W}$.

An analytic treatment of the one-loop effective potential was given in ref. [5]. In the presence of plasma effects a similar treatment of the potential is not available. Before performing the complete numerical analysis it is instructive to get an analytic feeling of the efectiveness of the screening provided by plasma effects. This can be done assuming that the bosonic contribution (6) to the effective potential (5) is dominated by one field, namely the $S$ field, and neglecting the contribution from the other bosons. The result can be used to illustrate other physical situations where the effective potential is dominated by one kind of bosonic fields 1 . The $\phi$ dependent part of the effective potential (5) can be written as

$$
V(\phi)=A(T) \phi^{2}+B(T) \phi^{4}+C(T)\left(\phi^{2}+K^{2}(T)\right)^{3 / 2}
$$

where

$$
\begin{gathered}
A(T)=-\frac{1}{2} \mu_{T}^{2}+\frac{1}{4}\left(\frac{\zeta^{2}}{3}+\frac{h_{t}^{2}}{2}\right) T^{2} \\
B(T)=\frac{1}{4} \lambda_{T} \\
C(T)=-\frac{\zeta^{3} T}{6 \pi} \\
K^{2}(T)=\frac{\left(\zeta^{2}+\lambda_{S}\right) T^{2}+3 M^{2}}{3 \zeta^{2}}
\end{gathered}
$$

and

$$
\begin{gathered}
\mu_{T}^{2}=\mu^{2}-\frac{\zeta^{2}}{8 \pi^{2}}\left\{m_{S}^{2}(v)+M^{2} \log \frac{c_{B} T^{2}}{m_{S}^{2}(v)}\right\}+\frac{3}{8 \pi^{2}} h_{t}^{2} m_{t}^{2}(v) \log \frac{m_{t}^{2}(v)}{c_{F} T^{2}} \\
\lambda_{T}=\lambda+\frac{\zeta^{4}}{8 \pi^{2}} \log \frac{c_{B} T^{2}}{m_{S}^{2}(v)}+\frac{3}{16 \pi^{2}} h_{t}^{4} \log \frac{m_{t}^{2}(v)}{c_{F} T^{2}}
\end{gathered}
$$

The temperature $T_{2}$ is defined by the condition $V^{\prime \prime}(0)=0$, or

$$
4 A^{2}-9 C^{2} K^{2}=0
$$

For $T<T_{2}$ the origin is a maximum, and there is a global minimum at $\phi \neq 0$ that evolves towards the zero temperature minimum. For $T>T_{2}$ the origin is a minimum and there is a maximum at $\phi_{-}(T)$ and a minimum at $\phi_{+}(T)$ given by

$$
\phi_{ \pm}^{2}(T)=\frac{1}{32 B^{2}}\left\{9 C^{2}-16 A B \pm 3|C| \sqrt{9 C^{2}+32\left(2 B^{2} K^{2}-A B\right)}\right\}
$$

At the temperature $T_{1}$ defined by the condition

$$
9 C^{2}+32\left(2 B^{2} K^{2}-A B\right)=0
$$

\footnotetext{
${ }^{4} \mathrm{~A}$ good example is the case of the minimal supersymmetric standard model [19] where the bosonic part of the effective potential can be dominated by the contribution of squarks.
} 
the maximum and minimum collapse $\phi_{-}\left(T_{1}\right)=\phi_{+}\left(T_{1}\right)$. For $T>T_{1}$ the origin is the only minimum.

Using (2831) the temperatures $T_{1}$ and $T_{2}$ can be written as

$$
\begin{gathered}
\zeta^{2} T_{1}^{2}=\frac{2 \lambda_{T_{1}}\left(\zeta^{2} \mu_{T_{1}}^{2}+\lambda_{T_{1}} M^{2}\right)}{\left(\frac{\zeta^{2}}{3}+\frac{h_{t}^{2}}{2}\right) \lambda_{T_{1}}-\frac{\zeta^{6}}{8 \pi^{2}}-\frac{2 \lambda_{T_{1}}^{2}}{3 \zeta^{2}}\left(\zeta^{2}+\lambda_{S}\right)} \\
T_{2}^{2}=\frac{1}{2 \alpha}\left\{\Lambda^{2}\left(T_{2}\right)+\sqrt{\Lambda^{4}\left(T_{2}\right)-16 \alpha \mu_{T_{2}}^{4}}\right\}
\end{gathered}
$$

where

$$
\begin{aligned}
& \alpha=\left(\frac{\zeta^{2}}{3}+\frac{h_{t}^{2}}{2}\right)^{2}-\frac{1}{3 \pi^{2}} \zeta^{4}\left(\zeta^{2}+\lambda_{S}\right) \\
& \Lambda^{2}(T)=\frac{1}{\pi^{2}} \zeta^{4} M^{2}+4\left(\frac{\zeta^{2}}{3}+\frac{h_{t}^{2}}{2}\right) \mu_{T}^{2}
\end{aligned}
$$

The nature of the phase transition depends on the relation between $T_{1}$ and $T_{2}$. For values of the parameters $\left(\zeta^{2}, \lambda_{S}, M\right)$ such that $T_{1}>T_{2}$ the phase transition is first order and the plasma screening is not very effective. When $T_{1}=T_{2}$ the phase transition becomes second order because the screening became more effective. In fact, the condition $T_{1}=T_{2}$ gives the turn-over from first to second order. It provides a surface in the space $\left(\zeta, \lambda_{S}, M\right)$ which separates first-order from second-order regions. The different regions in the $\left(\zeta, \lambda_{S}\right)$-plane are plotted in Fig.1 for $m_{h}=65 \mathrm{GeV}, m_{t}=120 \mathrm{GeV}$ and different values of $M$. An analytic approximation can be given, if one neglects loop corrections in $(32,33)$, as

$$
\frac{\zeta^{8}}{8 \pi^{2}}-\frac{2}{3} \lambda^{2}\left(\zeta^{2}+\lambda_{S}\right) \geq\left[\lambda\left(\frac{\zeta^{2}}{3}+\frac{h_{t}^{2}}{2}\right)-\frac{\zeta^{6}}{4 \pi^{2}}\right]\left(\frac{M}{v}\right)^{2}
$$

where the strict inequality corresponds to the subregion of the space $\left(\zeta, \lambda_{S}, M\right)$ for which the phase transition is first-order, and the equality corresponds to the turn-over to a second order phase transition. Since the right-hand side of eq. (41) is positive-definite ?, we can see from (41) how the parameters $M^{2}$ and $\zeta^{2}+\lambda_{S}$ in (4) and (5) influence the shielding of the first order phase transition. First, the larger the value of $M$ is, the easier one saturates the inequality in (41) and the easier one reaches a second-order phase transition. The same can be stated on $\lambda_{S}$, though its effect is damped by $\lambda^{2}$ and would become important only for a very heavy Higgs. For the same reason the effect of $\zeta$ is opposite, unless the Higgs is very heavy. These effects can be read off from Fig. 1 where no approximations are introduced.

The complete numerical analysis of eq.(5) is summarized in Figs.2-5 where we plot $\phi_{+}\left(T_{c}\right) / T_{c}$, where $T_{c}$ is the temperature at which the minima at $\phi_{+}$and at the origin are degenerate, for different regions of the space of parameters. In Figs.2 and 3 we plot $\phi_{+}\left(T_{c}\right) / T_{c}$

\footnotetext{
${ }^{5}$ It is easy to see that the necessary and sufficient condition for this turn-over to exist is that the righthand-side of eq. (41) is positive-definite. It is not a priori excluded that an isolated region exists in the space $\left(\zeta, \lambda_{S}, M\right)$ where the phase transition is second order. However we have checked that for phenomenological values of the parameters $\left(\lambda, h_{t}\right)$ this region does not exist.

${ }^{6}$ We have used, in Figs.2-5, the numerical computation of the integrals [6] giving rise to the hightemperature expansion of eqs. (77,8).
} 
versus $\zeta^{2}$ for $M=50 \mathrm{GeV}, 0 \leq \lambda_{S} \leq 1$ and $\lambda_{S}=0,0 \leq M \leq 1 \mathrm{TeV}$, respectively, and $m_{h}=65 \mathrm{GeV}, m_{t}=120 \mathrm{GeV}$. We can check from Fig.3 that for values of $M$ much greater than the electroweak scale, the $S$ field decouples and one recovers the MSM result.

For our choice of lagrangian parameters in (四), in particular for $M^{2}>0$, the sphaleron energy is minimized for field configurations with $S \equiv 0$ [20], which are the usual sphalerons in the MSM [2]. In that case the condition $E_{\mathrm{sph}}\left(T_{c}\right) / T_{c}>45$ [4] corresponds to $\phi_{+}\left(T_{c}\right) / T_{c}>$ $\left(\phi_{+}\left(T_{c}\right) / T_{c}\right)_{\min }$, which is plotted in Fig.4 (dashed line) versus $m_{h}$.

If we want to establish an absolute upper bound on the mass of the Higgs boson we need to optimize the phase transition with respect to the new parameters $\left(\zeta, \lambda_{S}, M\right)$. As can be seen from Figs.2 and 3 this is accomplished for $M=0$ and $\lambda_{S}=0$. As for $\zeta$, a quick glance at Figs. 2 and 3 shows that we should put it to its maximum value $\zeta_{\max }$. The usual requirement for $\zeta_{\max }$ is that the theory remains perturbative in all its domain of validity, from the electroweak scale to a high scale $\Lambda$. For that we have to study the renormalization group equations (RGE) of the minimal extension of the MSM provided by the lagrangian (1). At one-loop the only $\beta$-function of the MSM modified by the interactions of $S$ is $\beta_{\lambda}$ while there appear new $\beta$-functions for the new couplings $\lambda_{S}$ and $\zeta$, as [21]

$$
\begin{gathered}
\Delta \beta_{\lambda}=8 \zeta^{4} \\
\beta_{\lambda_{S}}=20 \lambda_{S}^{2}+8 \zeta^{4} \\
\beta_{\zeta^{2}}=\zeta^{2}\left[6 \lambda+6 h_{t}^{2}+8 \lambda_{S}+8 \zeta^{2}-\frac{3}{2}\left(3 g^{3}+g^{\prime 2}\right)\right]
\end{gathered}
$$

where we are using the convention

$$
16 \pi^{2} \frac{d x}{d t}=\beta_{x}
$$

for all couplings $x=\zeta^{2}, \lambda_{S}, \lambda, \ldots$. From eqs.43,44) we see that imposing $\lambda_{S}\left(M_{W}\right)=0$ as boundary condition, consistent with our previous requirement, we can reach the maximum value of $\zeta\left(M_{W}\right), \zeta_{\max }$, that will depend on $m_{h}, m_{t}$ and $\Lambda$. We have solved the system of RGE corresponding to the lagrangian (1) between $M_{W}$ and $\Lambda$ and obtained $\zeta_{\max }$ for different values of $m_{h}$ and $m_{t}$. The dependence of $\zeta_{\max }$ on $m_{h}$ is negligible for $60 \mathrm{GeV} \leq m_{h} \leq 100 \mathrm{GeV}$. In Table 1 we show $\zeta_{\max }^{2}$ for different values of $\Lambda$ and $m_{t}=90,120,175 \mathrm{GeV}$. In Fig.4 we plot $\phi_{+}\left(T_{c}\right) / T_{c}$ versus $m_{h}$ for $\lambda_{S}=0, \zeta=\zeta_{\max }$, as taken from Table 1 for the different values of $\Lambda, M=0$ and $m_{t}=120 \mathrm{GeV}$. To exhibit the dependence on $m_{t}$ we plot in Fig.5 $\phi_{+}\left(T_{c}\right) / T_{c}$ versus $m_{h}$ for $\lambda_{S}=0, M=0, m_{t}=90,120$ and $175 \mathrm{GeV}$, and $\zeta=\zeta_{\max }$, corresponding to $\Lambda=10^{6} \mathrm{GeV}$. In that case we see from Fig. 5 that avoiding baryon asymmetry washout imposes on the Higgs mass an upper bound of order $80 \mathrm{GeV}$.

In conclusion, we have obtained that the minimal extension of the MSM we have analyzed in this paper is consistent with $\Delta(B+L)$ and the experimental bounds on the Higgs mass provided the theory remains valid up to a scale $\Lambda \leq 10^{10} \mathrm{GeV}$. In the case the theory remains valid up to a higher scale (as e.g. the Planck scale) the former conditions would force some coupling constants to become non-perturbative below the high scale. Generically this would require extra physics between $\Lambda$ and the Planck scale.

\footnotetext{
${ }^{7}$ We are aware that values $M \ll v$ would require much more fine tuning than that required for the Higgs sector of the MSM. However we are taking $M=0$ only to establish an absolute upper limit on the Higgs mass.
} 


\section{References}

[1] G. t'Hooft, Phys. Rev. Lett. 37 (1976) 8; Phys. Rev. D14 (1976) 3432.

[2] F.R. Klinkhamer and N.S. Manton, Phys. Rev. D30 (1984) 2212; V.A. Kuzmin, V.A. Rubakov and M.E. Shaposhnikov, Phys. Lett. B155 (1985) 36; P. Arnold and L. McLerran, Phys. Rev. D36 (1987) 581; Phys. Rev. D37 (1988) 1020; A. Ringwald, Phys. Lett. B201 (1988) 510.

[3] For recent reviews, see: A.D. Dolgov, Kyoto preprint YITP/K-940 (1991); M.E. Shaposhnikov, preprint CERN-TH.6304/91; M. Dine, Santa Cruz preprint SCIPP 92/21; A. Linde, Stanford preprint SU-ITP-92-18.

[4] M.E. Shaposhnikov, JETP Lett. 44 (1986) 465; Nucl. Phys. B287 (1987) 757; Nucl. Phys. B299 (1988) 797; A.I. Bochkarev, S.Yu. Khlebnikov and M.E. Shaposhnikov, Nucl. Phys. B329 (1990) 493; A.I. Bochkarev, S.V. Kuzmin and M.E. Shaposhnikov, Phys. Lett. B244 (1990) 275; Phys. Rev. D43 (1991) 369.

[5] G.W. Anderson and L.J. Hall, Phys. Rev. D45 (1992) 2685.

[6] S. Weinberg, Phys. Rev. D9 (1974) 3357; L. Dolan and R. Jackiw, Phys. Rev. D9 (1974) 3320; D.A. Kirzhnits and A.D. Linde, Sov. Phys. JETP 40 (1975) 628; Ann. Phys. 101 (1976) 195.

[7] D.J. Gross, R.D. Pisarski and L.G. Yaffe, Rev. Mod. Phys. 53 (1981) 43; P. Fendley, Phys. Lett. B196 (1987) 175; J.I. Kapusta, Finite temperature Field Theory (Cambridge University Press, 1989).

[8] M.E. Carrington, Phys. Rev. D45 (1992) 2933.

[9] M.E. Shaposhnikov, Phys. Lett. B277 (1992) 324 and (E) B282 (1992) 483.

[10] D.E. Brahm and S.D.H. Hsu, Caltech preprints CALT-68-1762, HUTP-91-A064 and CALT-68-1705, HUTP-91-A063; C.G. Boyd, D.E. Brahm and S.D.H. Hsu, Caltech preprint CALT-68-1795, HUTP-92-A027, EFI-92-22.

[11] M. Dine, R.G. Leigh, P. Huet, A. Linde and D. Linde, Phys. Lett. B283 (1992) 319; Phys. Rev. D46 (1992) 550.

[12] P. Arnold, Phys. Rev. D46 (1992) 2628; P. Arnold and O. Espinosa, Washington preprint UW/PT-92-18, USM-TH-60.

[13] J.R. Espinosa, M. Quirós and F. Zwirner, Phys. Lett. B291 (1992) 115 and CERN preprint CERN-TH.6577/92, IEM-FT-58/92.

[14] W. Buchmüller, T. Helbig and D. Walliser, DESY preprint DESY 92-151.

[15] Particle Data Group, Review of Particle Properties, Phys. Rev. D45 (1992) S1. 
[16] M. Dine, P. Huet, R. Singleton Jr. and L. Susskind, Phys. Lett. B257 (1991) 351; M. Dine, P. Huet and R. Singleton Jr., Nucl. Phys. B375 (1992) 625.

[17] K. Enqvist, K. Kainulainen and I. Vilja, Phys. Rev. D45 (1992) 3415; N. Sei, I. Umemura and K. Yamamoto, Kyoto preprint NEAP-49 (1992).

[18] V. Jain and A. Papadopoulos, Berkeley preprint LBL-33067 (1992).

[19] G.F. Giudice, Phys. Rev. D45 (1992) 3177; S. Myint Phys. Lett. B287 (1992) 325.

[20] B. Kastening and X. Zhang, Phys. Rev. D45 (1992) 3884.

[21] T.P. Cheng, E. Eichten and L.-F. Li, Phys. Rev. D9 (1974) 2259. 


\section{Table captions}

Table $1 \zeta_{\max }^{2}$ for different values of $\Lambda$ and $m_{t}$.

\section{Figure captions}

Fig.1 Plots of the condition $T_{1}=T_{2}$, eqs.(37.38), in the $\left(\zeta, \lambda_{S}\right)$ plane for $m_{h}=65 \mathrm{GeV}$, $m_{t}=120 \mathrm{GeV}$ and values of $M$ from 0 to $300 \mathrm{GeV}$, with a step of $50 \mathrm{GeV}$. The lower (upper) curve corresponds to $M=0(M=300 \mathrm{GeV})$.

Fig.2 Plot of $\phi_{+}\left(T_{c}\right) / T_{c}$ as a function of $\zeta^{2}$ for $\lambda_{S}=0$ (upper curve), 0.5 and 1.0 (lower curve), $M=50 \mathrm{GeV}, m_{h}=65 \mathrm{GeV}$ and $m_{t}=120 \mathrm{GeV}$.

Fig.3 The same as in Fig.2, but for $\lambda_{S}=0, m_{h}=65 \mathrm{GeV}, m_{t}=120 \mathrm{GeV}$ and $M=0$ (upper curve), 50, 100, 150, 200, 250, 300, 350, 400 and $1000 \mathrm{GeV}$ (lower curve).

Fig.4 Plot of $\phi_{+}\left(T_{c}\right) / T_{c}$ versus $m_{h}$ for $\lambda_{S}=0, M=0, m_{t}=120 \mathrm{GeV}$ and $\zeta=\zeta_{\max }$, as taken from Table 1 for $\Lambda=10^{4}, 10^{6}, 10^{8}, 10^{10}, 10^{12}, 10^{14}$ and $10^{16} \mathrm{GeV}$.

Fig.5 The same as in Fig.4, but for $m_{t}=90,150$ and $175 \mathrm{GeV}, \Lambda=10^{6} \mathrm{GeV}$ and $\zeta_{\max }$ taken from Table 1. 


\begin{tabular}{|c|c|c|c|}
\hline \multirow{2}{*}{$\Lambda(\mathrm{GeV})$} & \multicolumn{3}{|c|}{$m_{t}(\mathrm{GeV})$} \\
\cline { 2 - 4 } & 90 & 120 & 175 \\
\hline \hline $10^{4}$ & 1.774 & 1.742 & 1.667 \\
$10^{6}$ & 1.095 & 1.067 & 1.011 \\
$10^{8}$ & 0.793 & 0.770 & 0.728 \\
$10^{10}$ & 0.624 & 0.604 & 0.573 \\
$10^{12}$ & 0.515 & 0.498 & 0.473 \\
$10^{14}$ & 0.439 & 0.424 & 0.405 \\
$10^{16}$ & 0.384 & 0.370 & 0.356 \\
\hline
\end{tabular}

\section{Table 1}

